\title{
A new model of Huntington's disease
}

Defects in oral communication are associated with many neurological disorders, including Huntington's disease. Despite their prevalence, the underlying causes of these communication deficits remain unclear. Using zebra finch song birds and transgenics, researchers at Rockefeller University (New York, NY) have established an important link between $m H T T$, a gene known to cause Huntington's disease in humans, and vocal communication (Nat. Neurosci. 18, 1617-1622; 2015).

Zebra finches are a popular model system for scientists studying language and communication. Zebra finches learn songs by mimicking the song of a 'tutor', or adult finch, similar to the way human children acquire language from their parents. Additionally, the brain circuitry required for vocal learning in songbirds is similar to that of humans. "There hasn't been a good animal model for any kind of speech disorder," stated lead author WanChun Liu in a press release. "Rats and mice, the most common lab animals, can't tell us much about speech disorders, since their vocalizations are innate, not learned. That's what makes songbirds so special." Liu and colleagues generated transgenic finches harboring $m H T T$ and examined the effects on vocal communication. By analyzing the songs of these mutant birds, they found that compared to wild-type zebra finches, the transgenic mutants displayed a notable defect in song learning.

Their songs contained incorrect syllables, long repeats and stuttering, and these defects worsened with age. These findings suggest that the neuronal circuits responsible for song learning were not intact in $m H T T$ mutants. To test this hypothesis, the authors created lesions in areas of the brain known to have a role in song learning. Although lesions to these brain circuits typically have no effect on singing in wildtype zebra finches, they caused significant impairments to song production in $m H T T$ zebra finches, suggesting that the song learning circuitry never correctly developed. Mutant songbirds also showed neuropathology similar to that seen in Huntington's disease, including decreased expression of

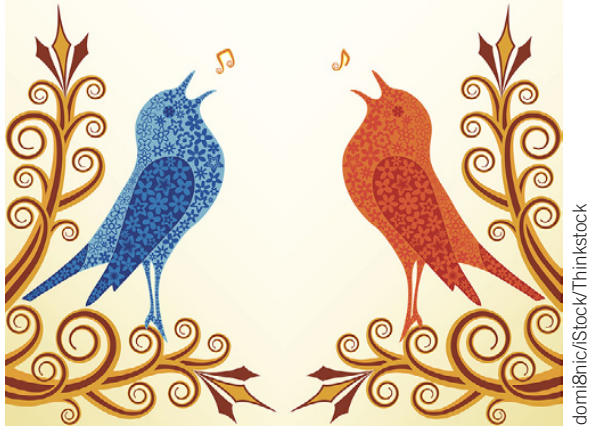

dopamine and androgen receptors and the presence of MHTT protein aggregates in the brain. The authors propose that these abnormalities could have contributed to the impaired song production.

Liu noted the far-reaching implications of this study: "Our work has relevance beyond Huntington's. By creating the first song bird models for vocal degeneration, we have achieved what I believe is a turning point for all kinds of diseases that affect speech, like Alzheimer's, Parkinson's, and autism."

Christine M. Scaduto

\section{REPAIR AND RESCUE FOR MUSCULAR DYSTROPHY}

Duchenne Muscular dystrophy (DMD) is a degenerative disease that afflicts young boys and is one of the most prevalent forms of muscular dystrophy. A defect in the gene for the cytoplasmic protein dystrophin leads to progressive loss in muscle function in these patients, cutting short their lives to a few decades. However, a recent study in a canine model of DMD by researchers at Boston Children's Hospital (Boston, MA) reveals that it might be possible to have this congenital defect and still lead a normal life (Cell 163, 1204-1213; 2015).

DMD is difficult to study, primarily owing to its rapid lethality and lack of animal models that mirror the symptoms seen in humans. Golden Retriever Muscular Dystrophy (GRMD) dogs, carrying the classic dystrophin mutation, make an excellent model system for DMD studies. These animals model the symptoms and disease progression seen in humans and typically have lifespans of only 2 years. In their study, Louis M. Kunkel and colleagues performed genetic analysis on two exceptional GRMD dogs that are able to walk, run and live a normal lifespan despite carrying the dystrophin mutation. Taking a closer look at these 'escaper' dogs, "We asked, what did the father pass to the escaper that made him able to escape the disease?"' says Natassia M. Vieira, first author of the study.

The answer lies deep in the genome of the escapers. Combining techniques such as genome-wide association studies and gene expression analysis, the scientists narrowed down the difference to a single nucleotide change in the gene Jagged 1 . This change, located in the promoter sequence of the gene, was present only in the escaper dogs and resulted in a heightened expression of Jagged 1 in their muscles. The muscles still underwent the same amount of wear and tear due to the dysfunction in dystrophin, but the higher Jagged1 levels improved the capacity for muscle repair. Engineering this change in a zebrafish model of DMD also led to a dramatic recovery of muscle function in the fish.

Most therapies for DMD focus on over-expressing dystrophin or its homologs in the patients. This study gives insight into the hitherto unexplored dynamics of muscle damage and repair at play in DMD. Manipulating Jagged1 activity in conjunction with gene therapy might open the doors to a longer and healthier life for DMD patients. 